\title{
Umweltmedizin und dermatologische Aspekte
}

\section{Environmental Medicine and Dermatological Aspects}

Autoren

Institut

\section{Trebing, C. C. Zouboulis}

Hautklinik und Immunologisches Zentrum, Städtisches Klinikum Dessau (Chefarzt: Prof. Dr. Christos C. Zouboulis)

\section{Bibliografie}

DOI $10.1055 / \mathrm{s}-2007-966083$

Akt Dermatol 2007; 33;

13-16 @ Georg Thieme

Verlag KG Stuttgart · New York ISSN 0340-2541

Korrespondenzadresse Dr. med. Dietrich Trebing Leitender Oberarzt Hautklinik und Immunologisches Zentrum, Städtisches Klinikum Dessau

Auenweg 38, 06847 Dessau dietrich.trebing@klinikumdessau.de

\section{Zusammenfassung \\ $\nabla$}

In Deutschland gelten ca. 32 Millionen Menschen als allergisch belastet, davon jedes 5 . Kind. Das allergologische Labor der Dessauer Klinik wurde im Zusammenhang mit der Gründung des Immunologischen Zentrums in Assoziation mit der Hautklinik 1986 gegründet und hat seither, dem raschen Erkenntnisgewinn auf dem Gebiet der

\section{Einleitung \\ $\nabla$}

Vor fast genau 100 Jahren, am 24. Juli 1906, wurde durch Clemens von Pirquet der Allergiebegriff in der Münchner Medizinischen Wochenschrift inauguriert. Der Haut als unmittelbar der Umwelt ausgesetztes, immunologisch aktives, größtes und am besten zugängliches Organ des menschlichen Körpers, kommt bei allergologischen Fragestellungen und Testungen eine besondere Rolle (,Indikatorfunktion“) zu.

Heute gelten in Deutschland ca. 32 Millionen Menschen als allergisch belastet, davon jedes 5 . Kind. Eine adäquate Therapie erfolgt nur bei ca. $10 \%$ der Patienten. Etwa 12 Millionen Menschen leiden an Heuschnupfen, 4 Millionen an einem Asthma bronchiale. Die Hauptallergene sind Pollen, Milben, Tierhaare und Nahrungsmittel [1]. Das allergologische Labor und die damit verbundene Sprechstunde wurden im Zusammenhang mit der Gründung des Immunologischen Zentrums in Assoziation mit der Hautklinik zur Mitte der 1980er Jahre durch Herrn Prof. Dr. H.-D. Göring in erweitertem Umfang an Diagnostik und Therapie gegründet und hat seither, dem raschen Erkenntnisgewinn auf dem Gebiet der Immunologie Rechnung tragend, eine beständige Weiterentwicklung und Erweiterung erfahren.

Im Rahmen der ambulanten und stationären Krankenversorgung werden Patienten aus dem Einzugsgebiet des Städtischen Klinikums der Re-
Immunologie Rechnung tragend, eine beständige Weiterentwicklung und Erweiterung erfahren. Das betrifft im Einzelnen die Abklärung und Behandlung bei Allergien der Haut und Schleimhäute, Inhalationsallergien, Medikamenten-, Nahrungsmittel-, Bienen- und Wespengiftallergien. Einen breiten Raum nehmen auch die Abklärung von Urtikariafällen, Pseudoallergien und Berufskrankheiten ein.

gion Anhalt/Dessau, aber auch weit darüber hinaus aus anderen Bundesländern behandelt. Für das Städtische Klinikum Dessau und umliegende Krankenhäuser werden zahlreiche konsiliarische Leistungen bei entsprechenden Fragestellungen erbracht.

Das betrifft im Einzelnen die Abklärung und Behandlung bei Allergien der Haut und Schleimhäute, Inhalationsallergien, Medikamenten-, Nahrungsmittel-, Bienen- und Wespengiftallergien. Einen breiten Raum nehmen auch die Abklärung von Urtikariafällen, Pseudoallergien und Berufskrankheiten ein. Weiterhin werden Autoimmun- und Immundefekterkrankungen der diagnostischen Klärung zugeführt und behandelt. Das diagnostische Repertoire des allergologischen Labors umfasst Epikutan- ( $\bullet$ Abb. 1), Abrisstest-, Prick-, Scratch- ( $\bullet$ Abb. 2) und Intrakutantest, Photopatchtest, die Rhinomanometrie ( Abb. 3) und Expositions- bzw. Provokationstestungen unter intensivmedizinischer Bereitschaft. Die durchgeführten Untersuchungen zur Hautphysiologie beinhalten die Bestimmung der Alkaliresistenz, des transepidermalen Wasserverlustes (TEWA-Meter, Abb.4), des Lipidgehaltes der Haut (Sebu-Meter, $\bullet$ Abb. 5) und den Nitrazingelbtest. Die Provokation mit physikalischen Reizen (Kälte, Wärme, Druck, UV-Strahlung) und cholinergische Testungen liefern Aussagen über die individuelle Empfindlichkeit und Veranlagung und lassen Rückschlüsse zur Auslö- 

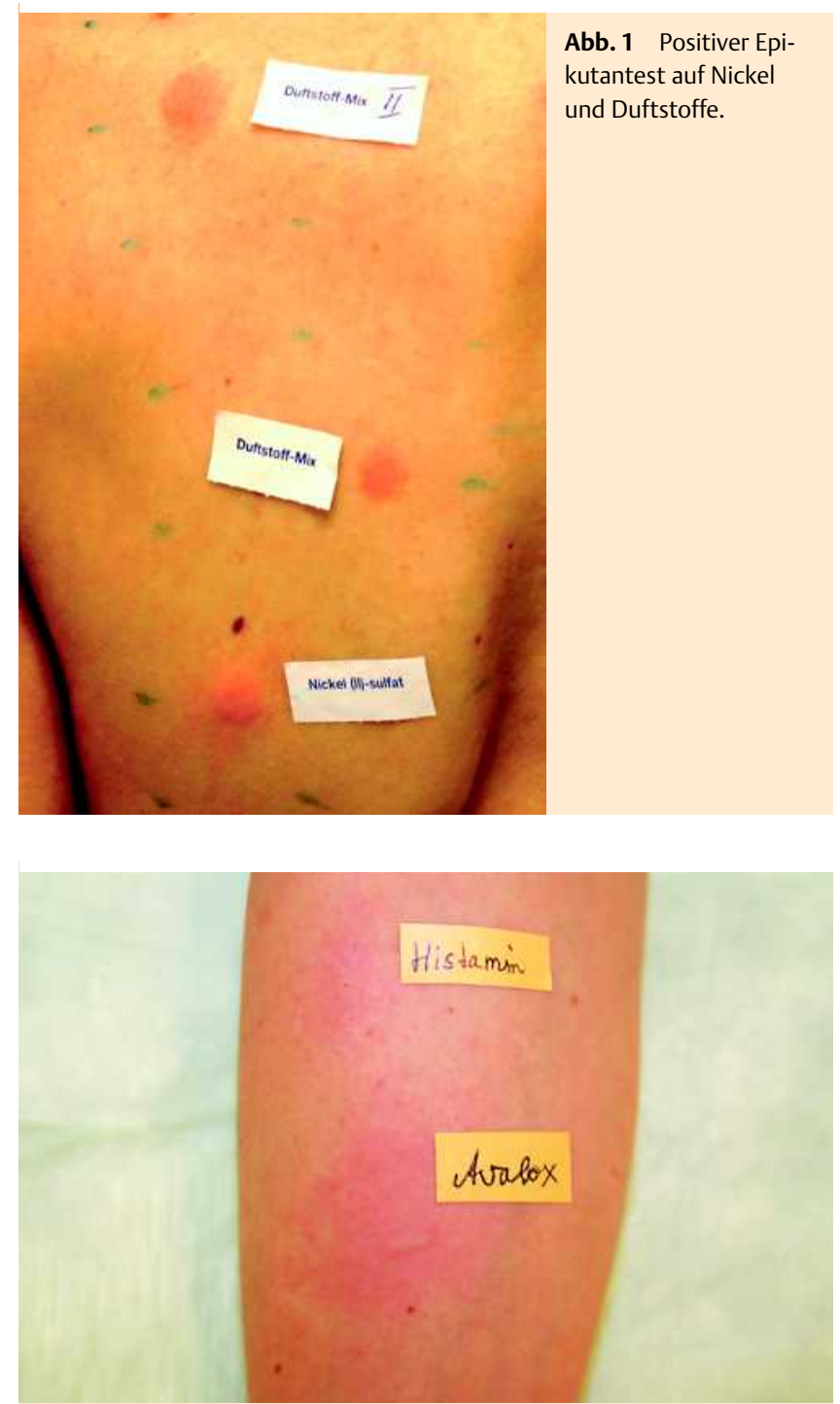

Abb. 2 Positiver Scratchtest auf ein Antibiotikum.

sung bestimmter Erkrankungen zu. Medizinische Begutachtungen erfolgen auf Anforderung von Berufsgenossenschaften, Unfallkassen und Sozialgerichten. Ebenfalls in den Laboratorien unserer Klinik durchgeführt werden sämtliche relevante In-vitroUntersuchungen, z. B. Bestimmungen des Gesamt- und spez. IgE, spez. IgG, zellulärer Antigenstimulationstest (CAST), Lymphozytentransformationstest (LTT), Auto-Antikörperdiagnostik. Im Folgenden einige exemplarische Ausführung zu einigen, häufig vorkommenden Allergien bzw. Pseudoallergien.

\section{Bienen- und Wespengiftallergien $\nabla$}

Jährlich ereignen sich in Deutschland ca. 10-20 Todesfälle durch Insektenstichreaktionen bei vermutlich hoher Dunkelziffer, systemische Reaktionen treten bei bis zu 5\% der Bevölkerung auf, gesteigerte Lokalreaktionen bei fast jedem 5 . Menschen. Bedenkt man, dass die Hyposensibilisierungstherapie mit Hymenopterengiften in über $90 \%$ der Fälle erfolgreich ist, unterstreicht dies die Wichtigkeit einer entsprechenden suffizienten Diagnostik und Behandlung [2].

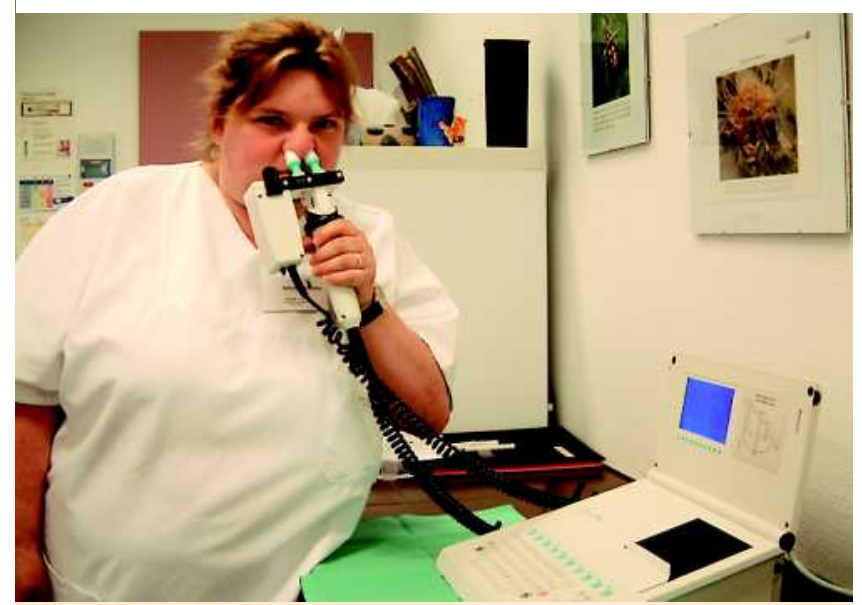

Abb. 3 Rhinomanometrie.

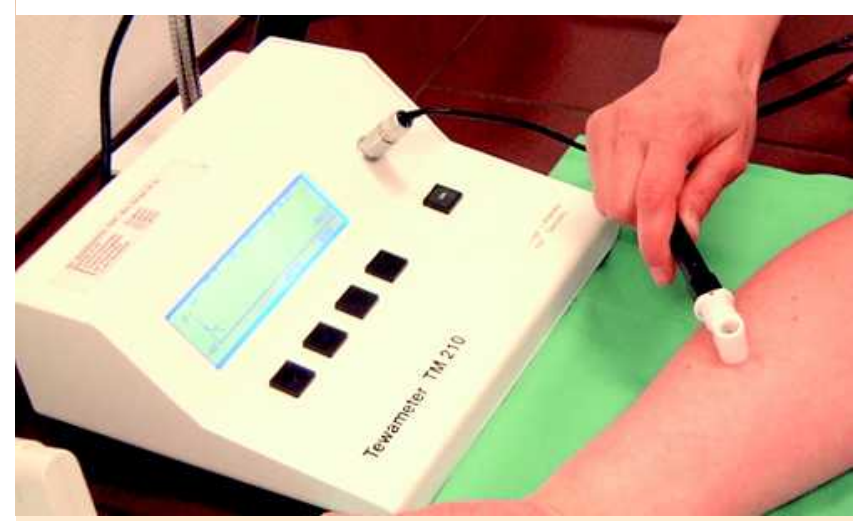

Abb. 4 Messung des transepidermalen Wasserverlustes (TEWA-Meter).

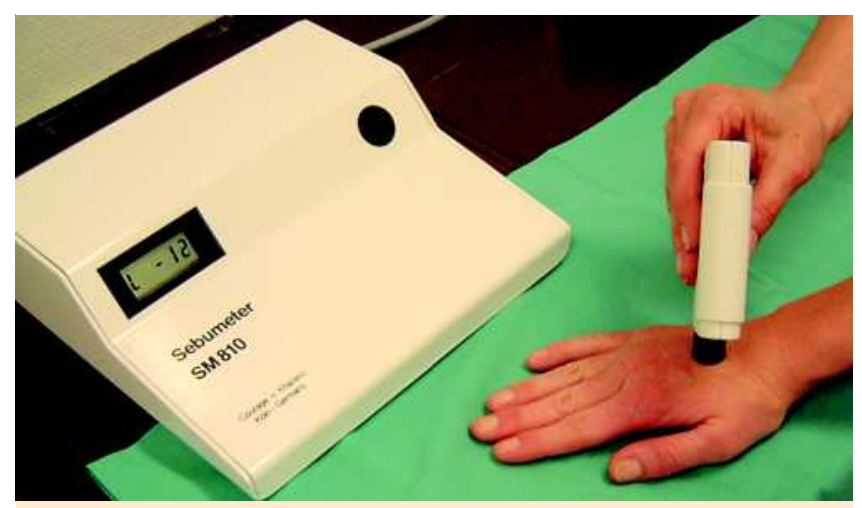

Abb. 5 Messung des Lipidgehaltes (Sebu-Meter).

Neben den anamnestischen Angaben dienen der Prick- und Intrakutantest und die Bestimmung des spez. IgE der Diagnosefindung, bedarfsweise auch unter Zuhilfenahme von zellulärem Antigenstimulationstest und Immunoblot. Die Stichprovokation wird, auch zur Therapiekontrolle, kontrovers beurteilt. 


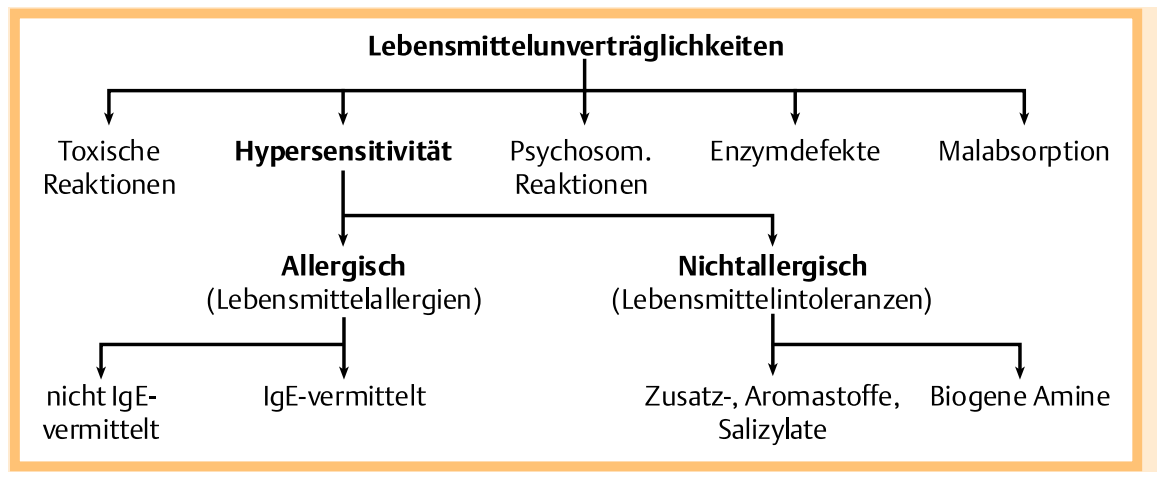

Abb. 6 Algorithmus der Lebensmittelunverträglichkeit.

Tab. 1 Häufigere Auslöser von Lebensmittelunverträglichkeiten

Glutenhaltiges Getreide und -erzeugnisse (Weizen, Roggen, Gerste, Hafer, Dinkel, Kamut)

Krebstiere und -erzeugnisse

Eier und -erzeugnisse

Fisch und -erzeugnisse

Erdnüsse und -erzeugnisse

Soja und -erzeugnisse

Milch und -erzeugnisse inkl. Laktose

Schalenfrüchte und -erzeugnisse (Mandel, Haselnuss, Walnuss,

Cashewnuss, Pecanuss, Paranuss, Pistazie)

Sellerie und -erzeugnisse

Senf und -erzeugnisse

Sesamsamen und -erzeugnisse

\section{Nahrungsmittelbedingte allergische und pseudoallergische Reaktionen \\ $\nabla$}

In der öffentlichen Wahrnehmung werden Nahrungsmittelunverträglichkeiten sehr häufig in ihrem Vorkommen überschätzt (20 - 30\% bei Schätzungen in der Bevölkerung), so dass der Allergiebegriff inzwischen inflationär gebraucht wird. Tatsächlich leiden nach Angaben der Lebensmittelsicherheitsbehörde der EU ca. 1-3\% der Erwachsenen und 4-6\% der Kinder an einer Lebensmittelallergie bzw. -intoleranz. Das zeigt auch hier die Wichtigkeit einer entsprechend fundierten Diagnostik, um zum einen die wirklichen, für den Patienten bedrohlichen Allergene zu identifizieren, zum anderen aber auch Klarheit zu schaffen, was vertragen wird und so den vermeintlich Betroffenen unnötige oder gar in Mangelzustände mündende Diäten zu ersparen [3].

Die Diagnostik umfasst auch hier die präzise Anamnese ( $\bullet$ Abb. 6), möglichst anhand eines Symptomtagebuches, Hautund Provokationstestungen und serologischen Untersuchungen. Während der Erwerb einer Allergie eine vorausgehende Sensibilisierungsphase erfordert und die Symptome in der Regel nicht dosisabhängig sind, können pseudoallergische Reaktionen ( $\odot$ Tab. 1) bereits beim Erstkontakt zu beobachten sein, dabei ist von einem Einfluss der Dosis auszugehen.

\section{Kontaktallergien \\ $\nabla$}

In der Allgemeinbevölkerung leiden zwischen 15 und 20\%(Frauen > Männer) im Laufe ihres Lebens an einem Kontaktekzem, ca. 7\% sind jährlich betroffen. Der Erwerb einer Sensibilisierung setzt einen vorangegangenen, mehr oder weniger langen und in-

\begin{tabular}{|l|}
\hline Tab. 2 Top 10 der Kontaktallergene \\
\hline Nickel (II)-sulfat \\
\hline Duftstoff-Mix \\
\hline Perubalsam \\
\hline Kobalt (II)-chlorid \\
\hline Kaliumdichromat \\
\hline Kolophonium \\
\hline p-Phenylendiamin \\
\hline Quecksilber (II)-amidchlorid \\
\hline Dibromdicyanobutan + 2-Phenoxyethanol \\
\hline Wollwachsalkohole \\
\hline
\end{tabular}

tensiven, zunächst reaktionslosen Kontakt mit dem entsprechenden Stoff voraus. Insgesamt besitzen Kontaktekzeme, auch durch die damit häufig verbundenen Berufskrankheiten eine große sozioökonomische Bedeutung [4].

Zu den Hauptallergenen ( $\odot$ Tab. 2) zählt Nickel (16,6\%), welches häufig bei jüngeren Menschen mit Kontakt zu Modeschmucks und Piercing zu Sensibilisierungen führt, aber auch z.B. in Brillengestellen, Reißverschlüssen vorkommt. Nicht allgemein bekannt ist, dass auch die perorale Nickelaufnahme mit der Nahrung (z. B. Kakao, Schokolade, Sojabohnen, Haferflocken, Nüsse, Mandeln, Hülsenfrüchte, nickelhaltige Kochgeschirre) bei einem Viertel aller Sensibilisierten zu Schüben ekzematöser Hautveränderungen führen kann.

An zweiter Stelle, mit über 1 Million Betroffenen in Deutschland, finden sich Duftstoffe und -mischungen. Sie sind gleichzeitig auch Aromastoffe und werden in Kosmetika, aber auch in Medikamenten, Reinigungsmitteln, technischen Flüssigkeiten und Lebensmitteln eingesetzt. Heute kennt man etwa 30000 Duftstoffe, wichtigste allergene Vertreter sind Eichenmoos (30\% d.F.) und Isoeugenol (20\%). Vor allem in den 1990er Jahren war es hierbei zu einem starken Anstieg gekommen.

Rang 3 nimmt der Duft- und Aromastoff Perubalsam ein, der auch in Medikamenten zur äußeren Anwendung vorkommt. Ähnlich dem Nickel kann es auch hier durch die Aufnahme über bestimmte Nahrungsmittel (z.B. Cola-Getränke, Vermouth, Fruchtgummis, Pralinen, Lakritz, Marzipan) und Tabakrauchen zu allergischen Reaktionen kommen [5].

Für die Diagnostik steht dem allergologisch ausgebildeten Arzt mit dem Epikutantest ( $\bullet$ Abb. 1) („Läppchentest“, „Patch-Test“) ein geeignetes Instrument zur Verfügung, welches eine Reproduzierbarkeit von $60-90 \%$ hat. 
Abstract

\section{Environmental Medicine and Dermatological Aspects $\nabla$}

Approx. 32 million people are regarded as allergic, among them every 5th child. The allergy laboratory of the Dessau Medical Center - founded 1986 - has been connected with the Department of Dermatology, undergone constant development and expansion taking the increasing knowledge in the field of immunology into account. This concerns the clarification and treatment of skin and mucosal allergies, inhalation allergies, drug, food, bees and wasp poison allergies. Urticaria, pseudoallergies and occupational diseases are often evaluated.

\section{Literatur}

1 Holzegel K. Gedächtnisvorlesung zu Ehren Marchioninis. Derm 2006; 12: $146-148$

2 Przybilla B, Rueff F, Fuchs T, Pfeiffer C, Rakoski J, Stolz W, Vieluf D. Insektengiftallergie - Leitlinie der Deutschen Gesellschaft für Allergologie und klinische Immunologie (DGAI). Allergo J 2004; 13: 186 - 190

3 Häberle $M$. Nahrungsmittelbedingte allergische und pseudoallergische Reaktionen. Der Deutsche Dermatologe 2006; 5: 316- 326

4 Schnuch A, Uter W, Geier J, Brasch J, Frosch PJ. Überwachung der Kontaktallergie: zur „Wächterfunktion“ des IVDK. Allergo J 2005; 14: $618-629$

5 Hausen BM. Rauchen, Süßigkeiten, Perubalsam - ein Circulus vitiosus? Akt Dermatol 2001; 27: 136-143

Buchbesprechung

Aloys Greither - Hautarzt zwischen Mozart, Hesse,

Dix und Scharl.

M. Irmisch

Norderstedt: Books on Demand, 2006. 64 S., 30 Abb., kart.

$13,80 €$

ISBN 3-8334-4909-8

Medizingeschichte ist ein Teil der Kulturgeschichte. Sie wird geprägt durch besondere Ereignisse und durch hervortretende Persönlichkeiten. Zu diesen gehört unzweideutig Aloys Greither (1913 - 1986), ein eigenwilliger, erfolgreicher Dermatologe und ein Kulturschaffender im besten Sinne des Wortes. Deswegen ist er beispielhaft herausgegriffen und hervorragend dargestellt in einer anmutigen und feinfühligen Broschüre von 64 Seiten, welche uns Maik Irmisch 2006 vorlegt. Sie ist aus seiner Doktorarbeit am Institut für Geschichte der Medizin in Dresden hervorgegangen. Die Betonung wird auf die kulturellen Leistungen von Aloys Greither gelegt, ohne seine Wertung als Dermatologe zu unterdrücken.

Aloys Greither hat wesentliche Bereiche von Kultur und Kunst sowohl aktiv ausführend befruchtet, wie auch verarbeitend, sammelnd oder beschreibend zu bereichern vermocht. Er spielte konzertreif Bratsche und Geige und er schrieb über Mozart, er zeichnete trefflich und sammelte moderne Kunst, er schrieb Romane und Gedichte und verkehrte mit Hermann Hesse sowie dem Autor des „Hauptmann von Köpenick“, Wilhelm Schäfer. Man findet gleichsam einen Querschnitt durch das kulturelle Leben seiner Zeit in Deutschland und den angrenzenden Staaten. Und er schrieb ein Buch über die Walser, deren er einer war, und erforschte die dort durch Inzucht angereicherten Erbleiden. Da viele die Haut betreffen, bildete sich daraus einer seiner dermatologischen Forschungsschwerpunkte, und er verglich Erbleiden, insbesondere Verhornungsstö- rungen und Keratosen der verschiedenen Alpentäler miteinander und mit denjenigen ähnlicher Isolate in ganz Europa. Er studierte und promovierte in München, erfuhr seine akademische Prägung bei Walther Schönfeld an der Universitäts-Hautklinik in Heidelberg, um nach einem halbjährigen Zwischenspiel in Aachen, 1962 in Düsseldorf die Nachfolge von HansTheodor Schreus anzutreten. Diesen prominenten Lehrstuhl hatte er mit Erfolg und Vehemenz bis zur Emeritierung 1982 inne. Im Jahre 1986 verstarb er im 73. Lebensjahr auf seinem Alterssitz in Holzhausen bei Bad Aiblingen.

Maik Irmisch schildert alle Facetten dieses besonders vielseitigen Kollegen mit Wärme, Liebe zum Detail und großem Einfühlungsvermögen. Er verfügt über viele Quellen und Dokumente aus der Familie, die er zu einer reichhaltigen und bunten Schilderung dieses wahrlich humanistisch zu nennenden Forschers und Künstlers fügt und mit 30 Abbildungen belebt.

Man liest die Broschüre mit Genuss und erfährt manches, das einem die Person und deren Zeit nahe bringt, man bewundert die enorme Breite und das Streben zur Tiefe und jeder, der noch das Glück hatte, Aloys Greither in seiner Lebensfülle zu erleben, erinnert sich seiner in Geschichten, Episoden, in Wort und Schrift, in Klängen und findet ihn immer wieder in vielen heute noch offenen Fragen. Lesen Sie dieses Buch und Sie werden überrascht sein, dankbar und bereichert.

E. G. Jung, Heidelberg 